\section{Iconic memory: Fallacies persist (?)}

ALISON BOWLING and WILLIAM LOVEGROVE University of Tasmania, Hobart, Tasmania 7001, Australia

Two recent papers (Coltheart, 1980; Long, 1980) have evaluated literature on the relationship between "iconic memory" and "visible persistence." In doing this, both writers focused on methods of measurement of these phenomena and the influence on them of luminance and duration. On the basis of his literature review, Coltheart (p. 210) concluded "the distinction between iconic memory and visible persistence is not merely terminological: they are actually different psychological processes." In comparison, Long concluded (p.814) that "the appealing parsimony of equating visible persistence and iconic memory, which has been the traditional view (e.g., Neisser, 1967), need not be abandoned." The present paper evaluates the strength of Long's arguments, and concludes that: (1) conflict arises over problems of definition; (2) the data from experiments using methods considered inappropriate by Long are very consistent and yield useful information about visible persistence; (3) it consequently cannot be claimed that the bulk of the evidence supports a positive relationship between stimulus intensity (or duration) and persistence duration; (4) it is misleading to claim that Long's data, usually collected under a specific combination of somewhat extreme experimental conditions, is representative of data in the area; and (5) iconic memory and visible persistence cannot be readily equated.

Long (1980) examined various methods of measuring visible persistence. Results of studies investigating luminance and/or duration effects were then evaluated from the consideration that some persistence measurement methods are more valid than others. Those methods regarded by Long as being less valid are considered to involve the measurement of effects in addition to persistence, or of phenomena that are only marginally related to it.

Methods thought to be most inappropriate include persistence-of-form studies (e.g., Bowling, Lovegrove, \& Mapperson, 1979; Meyer \& Maguire, 1977) and duration-of-stimulus procedures (e.g., Efron, 1970a, 1970b, 1970c; Haber \& Standing, 1970). The successive-field paradigm is considered to be the most effective, although different forms of this method also yield inconsistent results (Long, 1980, p. 810). Studies demonstrating an inverse persistence-intensity (or duration) relationship are rejected on the grounds that these studies employed the least valid methods. The remaining studies are considered to strongly support a positive relationship, and consequently the theory that iconic memory has a retinal basis. These conclusions are the opposite of those reached by Coltheart (1980), who considered the evidence for inverse persistence-intensity and persistence-duration relationships to be substantial, and that visible persistence is not the sole determinant of iconic memory.

\section{Definition Problems}

It is apparent that a major factor contributing to these opposite conclusions is a difference in the understanding of the meaning of the word "persistence." It has been clearly shown that there are at least two forms of persistence (Hawkins \& Shulman, 1979; Sakitt \& Long, 1979b), and it seems that Coltheart and Long each focus on a different one. One type of persistence is a visible response that continues beyond the stimulus offset and has a distinguishable termination (subjective offset). This is the Type I persistence of Hawkins and Shulman (1979). The other (Type II persistence) is a fading trace that is sometimes visible after the subjective offset and is similar to a weak afterimage (Coltheart, 1980; Sakitt, 1976b). To maintain the distinction between these two types of persistence, the terminology introduced by Hawkins and Shulman will be followed in this paper.

It is apparent that "visible persistence," as described by Coltheart (1980, p. 185), refers primarily to Type I persistence, whereas Long used the term "persistence" to mean Type II persistence (see pp. 788, 797). It also appears that while the majority of studies concerned with persistence (as opposed to iconic memory) are interested in Type I persistence, those conducted by Sakitt, Long, and their colleagues are primarily focused on Type II persistence. As will be discussed subsequently, the conditions employed by Sakitt and Long for most of their published work were optimal for the production and study of weak afterimages.

Consequently, Long's rejection of certain methods on the grounds that the phenomenon they measure may be only marginally related to persistence is based on the likelihood that they do not measure Type II persistence to any extent. This may be true, but does not justify their exclusion from the persistence literature, as they may be reasonably valid methods of investigating Type I persistence. Although all methods are subject to difficulties, as Long clearly establishes, the considerable consistency between the results of several methods justifies the conclusion that they do measure a phenomenon in common-probably Type I persistence. The discrepancy between the results obtained with different methods is thus partly due to the different properties of Type I and Type II per- 
sistence (Hawkins \& Shulman, 1979), and to the fact that one group of methods primarily investigates Type I, and another group, Type II.

\section{Effects of Intensity and Duration}

The considerable consistency between the results of various methods may be demonstrated by considering the effects of intensity and duration upon the result of the measurement. An inverse relationship between persistence and luminance has been demonstrated with the persistence-of-form method (Haber \& Standing, 1969; Bowling \& Lovegrove, Note 1), duration-of-stimulus procedures (Bowen, Pola, \& Matin, 1974; Efron, 1970c; Sakitt \& Long, 1979b), and an integration method for measuring the span of phenomenal simultaneity (Allport, 1968, 1970). Similarly, an inverse persistence-duration relationship has been demonstrated with many methods, including persistence of form (Bowling \& Lovegrove, 1980, 1981; Meyer \& Maguire, 1981), successive field paradigms (Di Lollo, 1977, 1980; Di Lollo \& Wilson, 1978; Hogben \& Di Lollo, 1974), and duration of stimulus (Bowen et al., 1974; Efron, 1970a, 1970b, 1970c). The shape of the persistence-duration relationship is also quite consistent across methods, with persistence initially declining linearly with increasing duration to a "critical duration" beyond which persistence is relatively constant or becomes negligible. This characteristic shape indicates that there may be a minimum duration of the response to a stimulus, and that Type I persistence is a result of any difference between response duration and stimulus duration (e.g., Di Lollo, 1977). Although the occurrence of persistence at longer stimulus durations may mean that additional components contribute to the persistence measure under some experimental conditions (Bowling \& Lovegrove, 1980, 1981; also Coltheart, 1980), this appears to elevate the overall measure rather than to affect the shape of the relationship between persistence and duration. Consequently, the consistent finding that persistence is related inversely to both luminance and duration with several experimental techniques is strong evidence for the validity of each of these techniques.

\section{Persistence-of-Form Method and Measures of Flicker}

One of the methods most strongly criticized by Long (1980) is the persistence-of-form method. Although this method is subject to criterion problems, these do not appear to be severe enough to affect its usefulness. Studies using the method have provided data similar to those of studies using other methods (e.g., Haber \& Standing, 1969, 1970). It is probable that differences in criteria between groups of subjects affect the overall measurement, but it is unlikely that criterion differences have a major influence on the results of different experimental con- ditions within subjects. Long's major criticism, however, is that it resembles methods used in studies of flicker perception, and since persistence (Type II) contributes little to flicker phenomena, the persistenceof-form method does not really measure persistence. This criticism is not valid for two reasons. (1) Long is primarily concerned with Type II persistence (afterimages) with properties very different from those of Type I persistence, which may be more closely related to other temporal phenomena such as flicker. The fact that Type II persistence may not contribute more than minimally to flicker perception is therefore irrelevant to the issue of whether the method is involved in the measurement of Type I persistence. (2) The fact that both methods involve the presentation of flickering stimuli does not mean that they measure the same phenomenon. With flicker studies, the dependent variable is the flicker rate or the stimulus luminance or contrast at which flicker is no longer detectable. Conversely, in the persistenceof-form method, the dependent variable is the maximum interval between successive cycles at which pattern is seen to be continuous. Under these conditions, flicker is almost always clearly present (e.g., Meyer \& Maguire, 1977). Since there is good evidence that flicker and pattern may be processed independently (e.g., Kulikowski \& Tolhurst, 1973), it cannot be assumed that the results of the two types of measurement are equivalent. Consequently, the method cannot be rejected on the basis of a superficial similarity to flicker fusion methods.

\section{Are Long's Data Representative of the Persistence Literature?}

With duration-of-stimulus procedures, it is possible under some conditions to measure the duration of both Type I and Type II persistence. The onset of a probe stimulus may be adjusted to coincide either with subjective offset or with the disappearance of any image elicited by the stimulus (Sakitt \& Long, $1979 \mathrm{~b})$. The effects of intensity on these two measures are opposite, the duration of Type I persistence decreasing and that of Type II persistence increasing with increasing luminance (Long, 1979; Sakitt \& Long, 1979b). The properties of Type I and Type II persistence are thus clearly different, and it appears that much of the confusion in the literature as to luminance and duration effects is due to a failure to clearly specify the type of persistence being measured. In addition, it is probable that some experimental conditions are more favorable than others for the production of Type II persistence, and on these occasions direct persistence-duration and persistence-intensity relationships may be obtained. In particular, the results obtained in successive-field paradigms appear to be particularly prone to influence by Type II persistence, since any stimulus spatial characteristics that are preserved in the afterimage 
will enhance the identification of the stimulus configuration. This paradigm is the one most favored by Long (1980) and the one used most frequently in his experimental work (Long \& Sakitt, 1980a, 1980b; Sakitt \& Long, 1978, 1979a).

The literature cited by Long in support of his argument for positive persistence-luminance and persistence-duration relationships consists of a series of studies by Sakitt, Long, and their colleagues and includes some studies using the partial report paradigm (e.g., Eriksen \& Rohrbaugh, 1970; Keele \& Chase, 1967; Sperling, 1960). The results of the partial report method may not be applicable to either Type I or Type II persistence, since the technique is primarily concerned with the investigation of iconic memory rather than with visible persistence (Coltheart, 1980). There appears to be a negligible, or very small, positive relationship between iconic memory and both luminance and duration. Furthermore, although some of the studies cited provide evidence for increased performance with increased energy levels, they do not provide any information about the relationship between luminance levels and iconic memory duration (e.g., Eriksen \& Rohrbaugh, 1970). The iconic memory studies he cites consequently do not provide support for Long's argument.

The remainder of the studies that support the argument were all performed by Long and his colleagues. The majority of these studies (regardless of method employed) were performed under experimental conditions that have rarely been employed in persistence studies. These included dark-adapted subjects, high luminance levels, presentation of bright stimuli against a dark background, and, in some cases, peripheral viewing. These conditions favor the production of afterimages (Brown, 1965). In view of the specific combination of experimental conditions employed by Long, it is hardly valid to claim that the data from these experiments represent general persistence phenomena.

Long and his colleagues have, however, recently performed some experiments using patterned stimuli on light backgrounds. These include Long and Beaton (1980b) and presumably Sakitt and Long (cited by Long \& Beaton, 1980a). In these experiments, also, the duration of Type II, rather than that of Type I, persistence is likely to be the dependent variable, since it is specified in Long and Beaton (1980b, p. 424) that "observers were instructed to attend to the fading trace of the target rather than to the offset of the target." The inverse relationship between persistence and spatial frequency referred to by Long and Beaton (1980a) may be a consequence of the reduction in the strength (and presumably also the duration) of the afterimages produced by $50-\mathrm{msec}$ presentations of higher frequency gratings (Corwin, Volpe, \& Tyler, 1976).

Coltheart (1980) has argued that the results of
Sakitt $(1975,1976)$ may be explained on the basis of weak afterimages produced by high stimulus intensities. Many of the results of the later papers by Sakitt, Long, and their colleagues may be similarly explained. Since the publication of Coltheart's paper, however, some of their publications report positive persistenceenergy relationships over a wide range of intensities, including very low ones. Specifically, Long and Sakitt (1980a) showed that increasing luminance over the range of .25 to $25 \mathrm{~cd} / \mathrm{m}^{2}$ and duration from 50 to $500 \mathrm{msec}$ improved performance on a task similar to that of Eriksen and Collins (1968). Long and Sakitt (1980b) obtained similar results with luminances ranging from approximately .32 to $320 \mathrm{~cd} / \mathrm{m}^{2}$ and durations ranging from 15 to $500 \mathrm{msec}$ in a task involving the detection of a spatial gap between two successively presented luminous rectangles. These data cannot entirely be explained by afterimages, since luminances of about $25 \mathrm{~cd} / \mathrm{m}^{2}$ or greater are necessary for the production of weak afterimages with dark-adapted subjects (Coltheart, 1980).

In these two papers, the dependent variable was the percentage of correct identifications for each combination of luminance and duration at a number of interstimulus intervals (ISIs). Thus, an improvement in performance with increasing stimulus energy was demonstrated. This is not surprising in view of the increased visibility of more intense, or longer, stimuli. The improvement in performance does not necessarily mean longer persistence, however, since the improvement was observed over all ISIs. To convincingly demonstrate longer persistence with these tasks, it would be necessary to demonstrate that the ISI at which the performance level for the longer and more intense stimuli asymptotes is longer than the asymptotic ISI for the weaker stimuli. Long and Sakitt's data do not clearly indicate that this is so. Figure 1 shows data from Long and Sakitt (1980b) replotted as a function of ISI. The graph shows data for G.L. (with the practiced 20-msec ISI) at the shortest and second largest stimulus durations (15 and $200 \mathrm{msec}$ ) and two relatively low luminance levels (.32 and $3.2 \mathrm{~cd} / \mathrm{m}^{2}$ ). It is evident that, although there is a clear improvement in performance with increased energy, there is no evidence for increased persistence. Consequently, these data do not provide any evidence for positive persistence-duration or persistenceluminance relationships when the luminances employed are too low to produce weak afterimages.

Stronger support for their argument may be seen in Figure 1 of Long and Sakitt (1980a). In this case, however, the data are given for only one subject, and a number of variables, including stimulus duration and number of trials per data point, are left unspecified. Higher energy levels again increase overall performance. Consequently, these data alone cannot be considered strong evidence for a positive persistenceluminance relationship. 


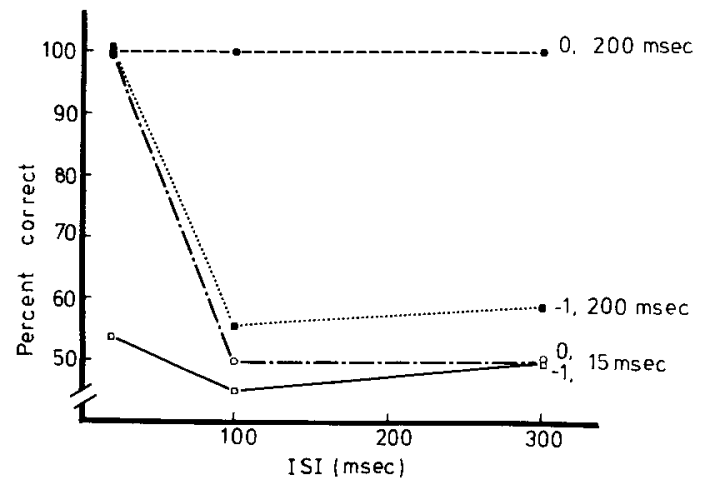

Figure 1. Data from Long and Sakitt (1980b) comprising two luminance levels $(-1$ and $0 \log \mathrm{mL}$ ) and two stimulus durations (15 and $200 \mathrm{msec}$ ), replotted as a function of ISI.

A general problem with measures of performance as a function of ISI, luminance, and duration on successive-field tasks is that performance may be influenced by factors other than persistence. It has already been shown how performance may increase with increasing energy apparently as a result of increased visibility rather than increased persistence duration. Other cues enhancing target identification become more readily discernible. When stimulus duration is manipulated, this problem may be overcome by equating stimulus energy over all durations. Under these conditions, an inverse relationship between persistence and duration is obtained (Di Lollo, 1977; Di Lollo \& Wilson, 1978; Hogben \& Di Lollo, 1974). Alternatively, a task of sufficient difficulty to eliminate all cues from either stimulus half (and from apparent movement differences, etc.) is necessary so that the item cannot be identified on any basis other than persistence. Neither the luminous rectangles nor the Eriksen and Collins forms of the successive-field paradigm appear to meet this criterion (Eriksen \& Collins, 1967, 1968; Long \& Sakitt, 1980a, 1980b). The dot matrix variation of the paradigm employed by Di Lollo and his colleagues may be more successful in this regard.

The material cited by Long (1980) consequently does not provide overwhelming support for the argument that persistence is directly related to both luminance and duration over a range of experimental conditions. Direct relationships are most frequently encountered under conditions in which weak afterimages are produced. It is these conditions which Long and his colleagues have used in their experimental work, and under these circumstances the properties of Type II persistence have been investigated. When luminance levels have been too low to produce afterimages, methodological problems and errors in interpretation appear to account for their conclusions. Consequently, it may be concluded that, while studies of Type II persistence may provide evidence for direct persistence-energy relationships under some conditions, the bulk of the literature, using several methods, consistently supports the existence of the inverse relationships for Type I persistence.

\section{Conclusions}

This paper has argued that there is considerable evidence for $a$ form of persistence (Type I) that is of relatively brief duration and that decreases in duration with both increasing stimulus luminance and duration. These effects have been consistently demonstrated with three or four different methods. The properties of this persistence are sufficiently different from those of iconic memory, as measured by the partial report technique, to conclude that Type I persistence contributes only minimally to iconic memory. A second form of persistence (Type II) has properties more similar to those of iconic memory, but this cannot account for the existence of iconic memory under all conditions (Coltheart, 1980). Consequently, although it may be more parsimonious to equate iconic memory and visible persistence, the available evidence does not strongly support such an identification, and it appears to be more accurate to consider that iconic memory and visible persistence result from different processes.

\section{REFERENCE NOTE}

1. Bowling; A., \& Lovegrove, W. Two components to visible persistence: Effects of luminance. Manuscript submitted for publication, 1982.

\section{REFERENCES}

Allport, D. A. Phenomenal simultaneity and the perceptual moment hypothesis. British Journal of Psychology, 1968, 59, 395-406.

Allport, D. A. Temporal summation and phenomenal simultaneity: Experiments with the radius display. Quarterly Journal of Experimental Psychology, 1970, 22, 686-701.

Bowen, R. W., Pola, J., \& Matin, L. Visual persistence: Effects of flash luminance, duration and energy. Vision Research, 1974, 14, 259-303.

Bowling, A., \& Lovegrove, W. The effect of stimulus duration on the persistence of gratings. Perception \& Psychophysics, 1980, 27, 574-578.

Bowling, A., \& Lovegnove, W. Two components to visible persistence: Effects of orientation and contrast. Vision Research, 1981, 21, 1241-1251.

Bowling, A., Lovegrove, W., \& Mappenson, B. The effect of spatial frequency and contrast on visual persistence. Perception, $1979,8,529-539$.

Brown, J. L. Afterimages. In C. H. Graham (Ed.), Vision and visual perception. New York: Wiley, 1965.

Colthen RT, M. Iconic memory and visible persistence. Perception \& Psychophysics, 1980, 27, 183-228.

Conwin, T. R., VolPE, L. C., \& TYLer, C. W. Images and afterimages of sinusoidal gratings. Vision Research, 1976, 16, 345-349.

Di LoLLo, V. Temporal characteristics of iconic memory. Nature, $1977,267,241-243$.

Dr LoLLo, V. Temporal integration in visual memory. Journal of Experimental Psychology: General, 1980, 109, 75-97. 
DI Lollo, V., \& WILson, A. E. Iconic persistence and perceptual moment as determinants of temporal integration in vision. Vision Research, 1978, 18, 1607-1610.

EFron, R. The relationship between the duration of a stimulus and the duration of a perception. Neuropsychologica, 1970, 8, 37-55. (a)

Efron, R. The minimum duration of a perception. Neuropsychologica, 1970, 8, 57-63. (b)

EFron, R. Effect of stimulus duration on perceptual onset and offset latencies. Perception \& Psychophysics, 1970, 8, 231-234. (c)

Eriksen, C. W., \& Collins, J. F. Some temporal characteristics of visual pattern perception. Journal of Experimental Psychology, 1967, 74, 476-484.

ERIKSEN, C. W., \& Collins, J. F. Sensory traces versus the psychological moment in the temporal organization of form. Journal of Experimental Psychology, 1968, 77, 376-382.

Eriksen, C. W., \& Rohrbaugh, J. Visual masking in multielement displays. Journal of Experimental Psychology, 1970, $83,147-154$.

Haber, R. M., \& Standing, L. G. Direct measures of short-term visual storage. Quarterly Journal of Experimental Psychology, $1969,21,43-54$.

Haber, R. M., \& Standing, L. G. Direct estimates of the apparent duration of a flash. Canadian Journal of Psychology, $1970,24,216-229$.

Hawkins, H. L., \& Shulman, G. L. Two definitions of persistence in visual perception. Perception \& Psychophysics, 1979, 25, 348-350.

Hogben, J. H., \& Di Lollo, V. Perceptual integration and perceptual segregation of brief visual stimuli. Vision Research, $1974,14,1059-1069$.

Keele, S. W., \& Chase, W. G. Short-term visual storage. Perception \& Psychophysics, 1967, 2, 383-386.

Kulikowski, J. J., \& Tolkurst, D. J. Psychophysical evidence for sustained and transient detectors in human vision. Journal of Physiology (London), 1973, 232, 149-162.

Long, G. M. Comment on Hawkins and Shulman's Type I and Type II visual persistence. Perception \& Psychophysics, 1979, 26, 412-414.

Long, G. M. Iconic memory: A review and critique of the study of short-term visual storage. Psychological Bulletin, 1980, 88, 785-820.
Long, G. M., \& Benton, R. J. The effects of spatial frequency and target type on perceived duration. Perception \& Psychophysics, 1980, 28, 413-421. (a)

Iong, G. M., \& Benton, R. J. The contribution of visual persistence to the perceived duration of brief targets. Perception \& Psychophysics, 1980, 28, 422-430. (b)

LoNG, G. M., \& SAKITT, B. The retinal basis of iconic memory: Eriksen and Collins revisited. American Journal of Psychology, 1980, 93, 195-206. (a)

Long, G. M., \& Sakitr, B. Target duration effects on iconic memory: The confounding role of changing stimulus dimensions. Quarterly Journal of Experimental Psychology, 1980, 32, 269285. (b)

Meyer, G. E., \& Maguire, W. M. Spatial frequency and the mediation of short-term visual storage. Science, 1977, 198, 524-525.

Meyer, G. E., \& Maguire, W. M. Effects of spatial frequency specific adaptation and target duration on visual persistence. Journal of Experimental Psychology: Human Perception and Performance, 1981, 7, 151-156.

Neisser, U. Cognitive psychology. Englewood Cliffs, N.J: Prentice-Hall, 1967.

SAKıт, B. Locus of short-term visual storage. Science, 1975, 190, 1318-1319.

SAkIT, B. Iconic memory. Psychological Review, 1976, 83, 257-276. (a)

SAKITT, B. Psychophysical correlates of photoreceptor activity. Vision Research, 1976, 16, 129-140. (b)

SakitT, B., \& Long, G. M. Relative rod and cone contributions in iconic storage. Perception \& Psychophysics, 1978, 23, 527-536.

SakitT, B., \& Long, G. M. Spare the rod and spoil the icon. Journal of Experimental Psychology: Human Perception and Performance, 1979, 5, 19-30. (a)

SakitT, B., \& Long, G. M. Cones determine subjective offset of a stimulus but rods determine total persistence. Vision Research, 1979, 19, 1439-1441. (b)

Sperling, G. The information available in brief visual presentations. Psychological Monographs, 1960, 74(11), 1-29.

(Manuscript received September 2, 1981; accepted for publication October 22,1981 .) 